\title{
Technical opportunities to extend the stoichiometric operation area of boosted gasoline engines in the high-volume segment
}

Dr. Claus Glahn, Co-authors: Dr. Ingo Hermann, Dr. Matthias Kluin, Achim Königstein, all Opel Automobile GmbH

Die Unterlagen wurden nicht zur Veröffentlichung freigegeben.

Wir bitten um Verständnis. 